\title{
Error Allocation in Complex Systems Design
}

\author{
Robert A. McDonald*
}

\begin{abstract}
A fidelity trade environment was conceived, formulated, developed, and demonstrated. This development relied on the advancement of enabling techniques including error propagation, metamodeling, and information management. These techniques were integrated with an existing commercial systems design framework and an intuitive graphical interface to create a fidelity trade environment.

A sensitivity approach to the propagation of error through complex systems was developed. This approach relied on the system sensitivity matrix to model the behavior of a complex system as a whole. In verification tests, the sensitivity approach provided approximate results substantially similar to a Monte Carlo approach that was many orders of magnitude more expensive. The rapid sensitivity approach to modeling error propagation enabled the responsive analysis required for an interactive environment.

In a case study, a notional transport aircraft was modeled in the fidelity trade environment. The system was decomposed and the fidelity trade environment was used to integrate the system. Then, a scenario was described where a decision maker used the fidelity trade environment at the beginning of a complex systems design problem. Using the environment, the designer was able to make design decisions while considering error and he was able to make decisions regarding required tool fidelity as the design problem continues. These decisions could not be made in a quantitative manner before the fidelity trade environment was developed.

The need for a new complex systems design technique was identified. A fidelity trade environment was conceived, establishing the need for advancement of three enabling techniques: error propagation, metamodeling, and information management. All of these techniques were integrated with an existing systems design architecture and an intuitive graphical interface, thereby creating the fidelity trade environment. This environment was applied to a representative complex system, thereby demonstrating its effectiveness in providing a new capability to the designer.
\end{abstract}

\section{Introduction \& Motivation}

Complex man-made systems are ubiquitous in modern technological society. The national air transportation infrastructure and the aircraft that operate within it, the highways stretching coast-to-coast and the vehicles that travel on them, and global communications networks and the computers that make them possible are all complex systems.

It is impossible to fully validate a systems analysis or a design process. Many systems are too large, complex, and expensive to build test and validation articles. Furthermore, the operating conditions throughout the life cycle of a system are impossible to predict and control for a validation experiment. Sometimes, designers are interested in revolutionary systems for which there is no historical counterpart which can be used for validation.

Error is introduced at every point in a complex systems design process. Every error source propagates through a complex system in the same way information propagates. If a system has feedforward, the errors feed forward. If a system has feedback, then errors feed back. If a system has coupled loops, then errors are coupled.

As with error propagation through a single analysis, error sources grow and decay when propagated through a complex system. These behaviors are made more complex by the complex interactions of a complete system. This complication and the loss of intuition that accompanies it makes proper error propagation calculations even more important to aid the decision maker.

\footnotetext{
*Assistant Professor, Aerospace Engineering, Cal Poly San Luis Obispo, AIAA Member
} 
Error can not be eliminated. However, it is possible to reduce many of the sources of error present in a complex systems analysis: better physical assumptions, fewer simplifications, better numerical implementations, and improved metamodels are all among the ways to reduce error at its source. Each error reduction has an associated cost while the complex system is varyingly sensitive to every error source. These relationships enforce a complex tradeoff between error sources. This tradeoff implies the ability to allocate an error budget associated with a complex system.

Error allocation and fidelity trade decisions answer questions like: Is the fidelity of a complex systems analysis adequate, or is an improvement needed? If an improvement is needed, how is that improvement best achieved? Where should limited resources be invested for the improvement of fidelity? How does knowledge of the imperfection of a model impact design decisions based on the model? How does this knowledge impact the choice and certainty of the design point? How does it impact the certainty of the performance of a particular design?

The creation of a fidelity trade environment relies on the support of enabling techniques from complex systems design. Among these enabling techniques are error propagation, metamodeling, and information management. These enabling techniques must then be combined into an intuitive interactive environment which may then be used by the decision maker.

Error propagation and allocation are fundamental yet often overlooked aspects of complex systems integration. Rather than comprehensively track and understand error throughout the system, error is frequently mitigated locally while its growth or decay through the system is ignored; in extreme cases, error is ignored entirely. Comprehensive tools for propagation and allocation of error will improve design fidelity and confidence, reduce design cost, and will provide guidance for investment in analysis capabilities.

\section{Error Propagation}

Error and uncertainty are ubiquitous in any complex systems study. As systems analysis and design techniques have progressed, there has been increasing interest and focus on the role of uncertainty in the system's life cycle and its impact on a robust design. Meanwhile, the role of error in the systems analysis and design processes has largely been neglected. In fact, the fundamental difference between error and uncertainty is not frequently recognized. This research does not focus on robust design, uncertainty propagation, error identification, or error quantification. Instead, this research focuses on error propagation for the purpose of error allocation and fidelity trades.

Any discussion of the use of tools in engineering should include a discussion of the related concepts of fidelity, error, validation, and verification. Roache ${ }^{1}$ establishes technical definitions for the concepts of validation and verification in the context of analysis tools. In his structure, validation is a process of ensuring that one is solving the right equations and verification is a process of ensuring that one is solving the equations right.

In this research, the term fidelity is used as a measure of error. A tool with higher fidelity is defined to have less error. In this context, a judgement of fidelity has nothing to do with the sophistication of the tool, only of the error in the result. A validation exercise can be interpreted as quantifying the fidelity or error of a tool and then making a decision as to whether that level of fidelity is appropriate for the problem at hand. In this manner, a fidelity trade environment is a decision making tool for systems analysis validation.

In addition to error inherent to a calculation, in any calculation the error in any input quantity contributes to an erroneous output. The magnitude of error in an output may be diminished or amplified relative to the error of the inputs. In a series of calculations representing a complex system, error can build up and interact in non-intuitive ways. The behavior of error becomes even more obscure when the series of calculations are coupled.

Error propagation is a set of techniques used to quantify and understand the error in an output quantity based on some knowledge of the error of the input quantities and error introduced during the process. Error propagation was originally developed for the experimental sciences. ${ }^{2,3}$ In an experiment, the source of all error is measurement, as Mother Nature makes no mistakes. Conversely, computer analysis codes have many sources of error. They are subject to error in their inputs and their process but there is no measurement error in their outputs., ${ }^{4,5}$

Error propagates through every component of a complex system. Understanding the error propagation through a complex system starts with understanding error propagation through an individual component. The JMP statistics and data analysis software recently added the capability to propagate error through 
individual components using techniques similar to those used in this research. ${ }^{6}$ Of course, few complex systems can be adequately modeled with a single black box. Because of the interaction and flow of information through a complex system, the error propagation through a complex system is necessarily more complicated.

As with error propagation through a single analysis, error sources grow and decay when propagated through a complex system. These behaviors are made more complex by the complex interactions of a complete system. This complication and the loss of intuition that accompanies it makes proper error propagation calculations even more important to aid the decision maker.

The complex system is modeled as a system of equations that must be iterated to achieve compatibility. This iteration greatly increases the expense of exploring a design space. Re-converging a complex system in this manner to investigate the impact of a potential error source would be very expensive. Instead, error propagation is combined with system sensitivity analysis and matrix inversion to instantly propagate an error through the complex system without re-converging the system.

Although the notation and approach to error propagation used here are different from those of Du and Chen, ${ }^{7}$ the resulting equations are equivalent.

\section{II.A. System Description}

A coupled system of systems, no matter how complex, may be described as a functional black box, $\mathbf{f}(\mathbf{x})$, where $\mathbf{f}$ is a vector of $m$ functions of $\mathbf{x}$, a vector of $n$ variables.

The system, $\mathbf{f}$, is broken into $l$ subsystems, $\mathbf{f}=\mathbf{g}_{i} ; i=1, \ldots, l$, each a vector of $m_{i}$ functions $\mathbf{g}_{i}=$ $g_{i, j} ; j=1, \ldots, m_{l}$ of $\mathbf{x}$ and the other subsystems; this is written $\mathbf{g}_{i}\left(\mathbf{x}, \mathbf{g}_{k \neq i}\right)$. Here, the comma notation $g_{i, j}$ is used to mean the $j$ th element of vector $\mathbf{g}_{i}$, it does not imply that $\mathbf{g}$ is a matrix, nor does it imply differentiation with respect to $j$. The total number of functions in the system, $m$ is equal to the sum of the number of functions in each of the subsystems $m_{i}$. i.e. $m=\sum_{i=1}^{l} m_{i}$

\section{II.B. Total Differential}

The total differential of the subfunctions may be written as Equation 1. This equation intuitively represents the influence of the coupled components on one another. If one quantity were to be infinitesimally perturbed in some way, Equation 1 would predict the influence of that perturbation on the entire system. The first term on the right hand side of Equation 1 accounts for the impact of a perturbation to a quantity on that quantity while the second term accounts for the impact of the perturbation to the quantity due to the interactions with all the other components. Unfortunately, this equation is difficult to apply because total differential terms appear on both sides of the equation. This equation says nothing about the cause or source of the perturbation.

$$
d \mathbf{g}_{i}=\partial \mathbf{g}_{i}+\sum_{j=1, j \neq i}^{l} \frac{\partial \mathbf{g}_{i}}{\partial \mathbf{g}_{j}} d \mathbf{g}_{j}
$$

The differential terms $d \mathbf{g}_{i}$ and $\partial \mathbf{g}_{i}$ are both $m_{i} \times 1$ vectors. Whereas the sensitivity term, $\frac{\partial \mathbf{g}_{i}}{\partial \mathbf{g}_{j}}$, is a $m_{i} \times m_{j}$ vector.

Similarly, the definition of the total differential of the subfunctions when subjected to arbitrary perturbations in $\mathbf{x}$ yields Equation 2.

$$
d \mathbf{g}_{i}=\frac{\partial \mathbf{g}_{i}}{\partial \mathbf{x}} d \mathbf{x}+\sum_{j=1, j \neq i}^{l} \frac{\partial \mathbf{g}_{i}}{\partial \mathbf{g}_{j}} \frac{d \mathbf{g}_{j}}{d \mathbf{x}} d \mathbf{x}
$$

\section{II.C. System Sensitivity Analysis}

System sensitivity analysis (SSA $)^{8,9}$ is a technique developed for multi-disciplenary optimization (MDO) which finds equal applicability to the propagation of error in complex systems. SSA addresses the difficulty outlined in the previous section, that the total differential of a component depends on the total differentials of all the other components in the system. Equation 1 may be rearranged to the following form.

$$
\partial \mathbf{g}_{i}=d \mathbf{g}_{i}-\sum_{j=1, j \neq i}^{l} \frac{\partial \mathbf{g}_{i}}{\partial \mathbf{g}_{j}} d \mathbf{g}_{j}
$$


This equation may be written in matrix form as follows.

$$
\partial \mathbf{f}=\left[\begin{array}{cccc}
\mathbf{I}_{1} & -\frac{\partial \mathbf{g}_{1}}{\partial \mathbf{g}_{2}} & \cdots & -\frac{\partial \mathbf{g}_{1}}{\partial \mathbf{g}_{l}} \\
-\frac{\partial \mathbf{g}_{2}}{\partial \mathbf{g}_{1}} & \mathbf{I}_{2} & & \vdots \\
\vdots & & \ddots & -\frac{\partial \mathbf{g}_{l-1}}{\partial \mathbf{g}_{l}} \\
-\frac{\partial \mathbf{g}_{l}}{\partial \mathbf{g}_{1}} & \cdots & -\frac{\partial \mathbf{g}_{l}}{\partial \mathbf{g}_{l-1}} & \mathbf{I}_{l}
\end{array}\right] d \mathbf{f}
$$

The matrix in the above equation is the system sensitivity matrix, $\mathbf{M}$, which is $m \times m$. As expected, $\partial \mathbf{f}$ and $d \mathbf{f}$ are both $m \times 1$ and $\mathbf{I}_{i}$ is an $m_{i} \times m_{i}$ identity matrix. Using this definition, the equation may be compactly written as shown below.

$$
\partial \mathbf{f}=\mathbf{M} d \mathbf{f}
$$

Inversion of the system sensitivity matrix isolates all total differential terms on the left hand side of the equation as shown below in Equation 3.

$$
d \mathbf{f}=\mathbf{M}^{-1} \partial \mathbf{f}
$$

This is an equation for the total differential of a coupled system of equations in terms of only partial differentials. This important result addresses the difficulty encountered in the previous section, significantly improving the ease, accuracy, and cost of the calculation of a total differential.

The system sensitivity matrix $\mathbf{M}$ completely describes component interactions. Upper-triangular terms represent feedback while lower-triangular terms represent feedforward. Zero terms indicate independence while large terms indicate strong dependence. Visualization of the sensitivity matrix yields a result substantially similar to a Design Structure Matrix (DSM) diagram. ${ }^{8,10}$

As expected, the impact on a system subject to perturbations in components and input values is simply the sum of the impact due to individual perturbations as shown in Equation 4.

$$
d \mathbf{f}=\mathbf{M}^{-1}\left(\partial \mathbf{f}+\frac{\partial \mathbf{f}}{\partial \mathbf{x}} \partial \mathbf{x}\right)
$$

\section{II.C.1. Application to Finite Increments}

The exact differential statement expressed in Equation 4 may be applied in an approximate manner for finite increments as in Equation 5; this amounts to a linearization of the exact equation and is appropriate for small increments.

$$
\Delta \mathbf{f} \approx \mathbf{M}^{-1}\left(\delta \mathbf{f}+\frac{\partial \mathbf{f}}{\partial \mathbf{x}} \Delta \mathbf{x}\right)
$$

Perturbations may be introduced to Equation 5 to perform a variety of sensitivity studies. Depending on the interpretation of the perturbations, one may perform simple sensitivity studies, obtain the direction of improvement for a system-level optimizer, model the impact of a determinate error, or of a bounded error source.

\section{II.D. Indeterminate Error}

Instead of making a worst-case assumption about the behavior of error as done with bounded error, the error source may be treated comprehensively as a random variable. Application of the definition of the variance of a random variable to the system perturbation results in the following relation for the variance of the system perturbation.

$$
\sigma_{\mathbf{f}}^{2}=\frac{1}{N-1} \sum(\Delta \mathbf{f})^{2}
$$

The use of Equation 6 as a vector equation is noteworthy: the square operation is carried out in a term-by-term sense, not in a vector sense. Substitution of Equation 5 into this equation yields the following relation; only the first term of Equation 5 is used here for clarity. 


$$
\sigma_{\mathbf{f}}^{2} \approx \frac{1}{N-1} \sum\left(\mathbf{M}^{-1} \delta \mathbf{f}\right)^{2}
$$

This expression is approximate only in the sense that Equation 5 is a linearized approximation of the exact impact of finite increments on the system.

The square operation is carried out on Equation 7, and the variance $\left(\varsigma_{\mathbf{f}}\right)$ and covariance $\left(\varsigma_{f_{j} f_{k}}\right)$ of the unpropagated perturbations is recognized, resulting in the following expression.

$$
\sigma_{\mathbf{f}}^{2} \approx\left(\mathbf{M}^{-1}\right)^{2} \varsigma_{\mathbf{f}}^{2}+\left[\left(M^{-1}\right)_{i j}\left(M^{-1}\right)_{i k} \varsigma_{f_{j} f_{k}}^{2}\right]_{j \neq k}
$$

When any two perturbations, say $\delta f_{j}$ and $\delta f_{k}$, are uncorrelated, their covariance is identically zero $\left(\varsigma_{f_{j} f_{k}} \equiv 0\right)$. Any two unpropagated errors will be independent random variables and therefore uncorrelated. Consequently, in the context of error propagation, the second term may be dropped; this results in Equation 8 for the propagation of indeterminate error. Recall that the square operation on the vector and matrix terms is done on a term-by-term basis.

$$
\sigma_{\mathbf{f}}^{2} \approx\left(\mathbf{M}^{-1}\right)^{2} \varsigma_{\mathbf{f}}^{2}
$$

The derivation of Equation 8 demonstrates a Pythagorean sum as a technique for combining independent random variables. In the same way, a Pythagorean sum may be used to combine any independent sources of indeterminate error. Straightforward application of a Pythagorean sum to Equation 5 results in the following equation for the propagated indeterminate error of a system subject to error in components and inputs.

$$
\sigma_{\mathbf{f}}^{2} \approx\left(\mathbf{M}^{-1}\right)^{2}\left[\begin{array}{lll}
\varsigma_{\mathbf{f}}^{2}+\frac{\partial \mathbf{f}}{\partial \mathbf{x}} & \sigma_{\mathbf{x}}^{2}
\end{array}\right]
$$

This equation may be used to track the propagation of error throughout an arbitrarily complex system. Multiple sources of component error, say physical fidelity, numerical convergence, and metamodel approximation, may all be handled through an appropriate Pythagorean sum. The same approach can be used for multiple sources of input error.

The assumption of the independence of separate error sources, which allows the covariance term to be dropped, deserves further comment. It is essentially a statement that when one source of error is positive and large, nothing can be said about the magnitude or sign of another error source. For example, if a drag estimation significantly overestimates the drag on a vehicle, nothing conclusive can be said about the error in a weight estimation of the same vehicle.

\section{Fidelity Trade Environment}

The systems visualization environment is the centerpiece of the fidelity trade environment. It is built upon the data repository, metamodel, and other components, and provides the systems model and error analysis as well as the decision making interface for error and design. The dynamic interactive environment has been developed to display a large quantity of multivariate information in a format intuitive to the designer. Additionally, the display can be re-configured easily by the user to meet the demands of any particular problem. A typical view of the interactive environment was included as Figure 1.

On the left hand side, a tabbed window allows the designer to choose how he wishes to interact with the interface. A designer may set system level inputs, introduce and set various sources of error, or add and adjust constraints on the design space. In the included example, the designer is interacting with various sources of error. These errors are propagated throughout the complex system and their impact is depicted throughout this environment.

Across the top, a bar graph reports the error of each of the output quantities at the current design point. Each bar is built up from the various propagated error contributions. Using this feature, the designer can quickly assess the heavy-hitting error sources at the design point.

At the center of the right side, a design structure matrix (DSM) diagram depicts the structure of the complex system. Interaction with the DSM reveals the specific quantities communicated between the contributing analyses. 


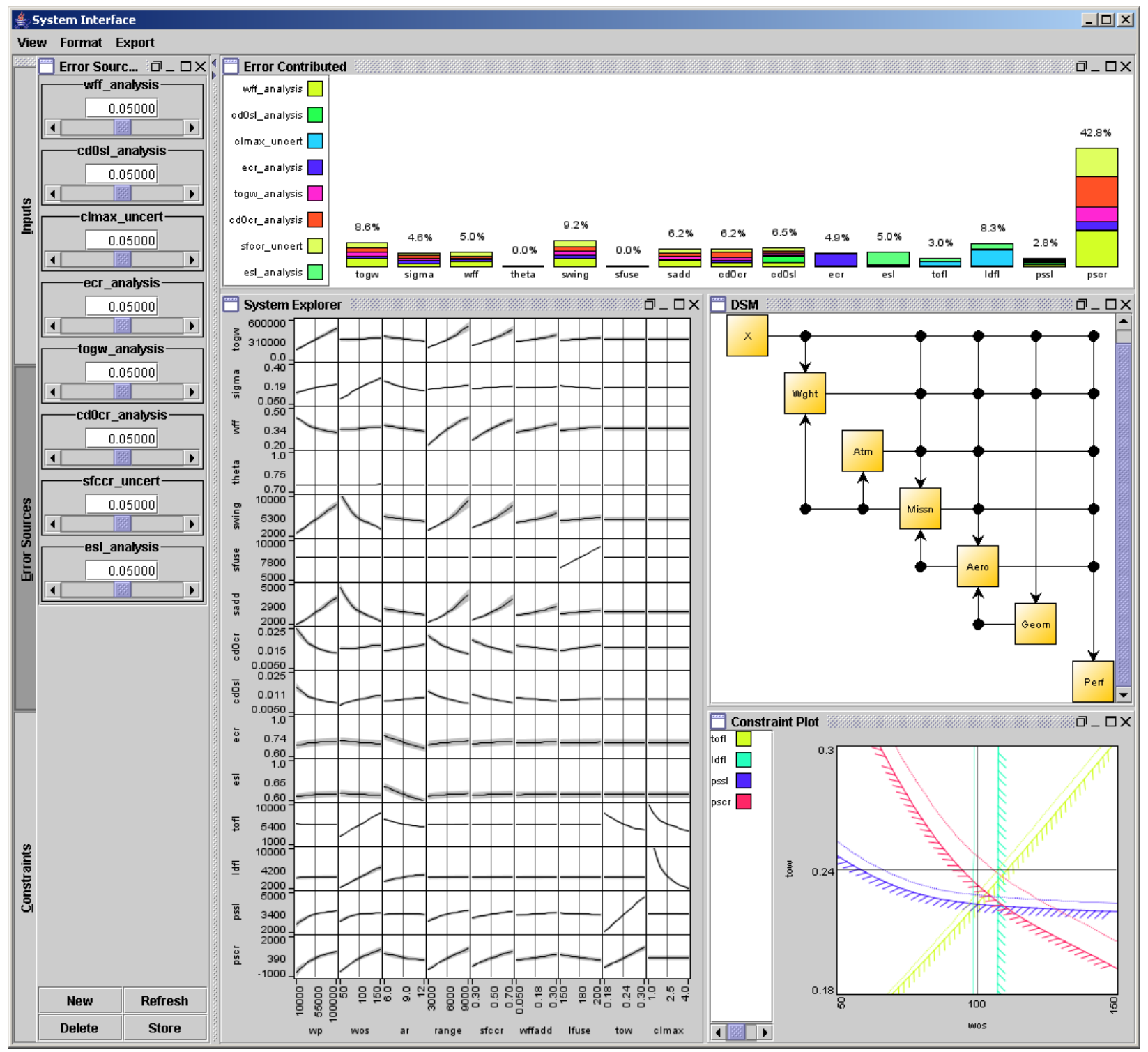

Figure 1. Fidelity trade environment.

In the center, a system explorer allows the designer a quick overview of the behavior of the design space. Each column corresponds to a system level design variable and each row corresponds to a output quantity. The system is re-converged for every point in this display. The designer can interact with the system by dragging hairlines that define the current design point. This depiction of system behavior has been augmented with a shaded band to indicate the propagated error on every quantity throughout the design space.

Finally, in the bottom right corner, a constraint diagram depicts a view of the design space with various constraints applied to the output quantities. The constraints are each drawn as a colored line, with a hatched profile depicting the constrained region. Once again, all of the error sources have been propagated throughout the system, and their impact on the location of the constraint is indicated by a matching dotted line. This allows the designer to understand not only how the design point must be moved to account for error, but also how which constraints are active in determining the design point can change due to error.

This environment exists as a part of a larger distributed design environment (DDE). ${ }^{11}$ At the core of this DDE lies a database used to manage information about design studies, analysis tools, system architecture, system error, metamodeling, the results of all analysis runs, and more. This DDE has been developed to use 
Phoenix Integration's Analysis Server ${ }^{12}$ protocol to integrate seamlessly with existing design environments. The DDE also uses an advanced Gaussian Process metamodel as a surrogate to facilitate error propagation and design space visualization. Because of the integrated database, the user is not faced with the burden of maintaining the analysis runs used to train the Gaussian Process. Furthermore, because of the tight integration with Analysis Server, the user may use the resulting Gaussian Processes as a simple drop-in component to Model Center and other systems design tools.

\section{Example Complex System}

In order to test and demonstrate the fidelity trade environment, a complex system must be modeled and exercised. The test system must have complexities representative of typical complex systems. The fidelity trade environment must then be exercised in a manner which demonstrates the use of the fidelity trade environment by a decision maker. The experiment not only needs to demonstrate that the ideas and method embodied by the fidelity trade environment are possible and correct, but that the environment proves useful for the decision maker.

Systems design techniques are applicable to any system at any level of abstraction. This allows the designer to tailor the level of abstraction to the problem at hand. In demonstrating the utility and correctness of a systems design technique, it is not very important what specific system is considered. It is important that the example system exhibit challenges and traits representative of complex systems. For this research, a wide-bodied transport aircraft was chosen as the demonstration system. As a clear example of a complex system, the transport aircraft takes a pivotal role in a system of systems hierarchy present in everyday life.

This complex system model is made of a series of tasks, each pertaining to a classical discipline of aerospace engineering. Each task defines an interface, a set of inputs and outputs. Any analysis which implements the task interface may be used in the system study. Simple implementations of the task interfaces were developed as prototype analyses. The fidelity trade analysis can then be used to identify which task implementations must be improved. Gaussian process metamodels were fit to the prototype analysis implementations over a range of input variables appropriate for the example problem. Although the discussion here is necessarily brief, this example has been fully documented by the author. ${ }^{11}$

The complex system model representing an aircraft was assembled from simple tasks representing the primary disciplines contributing to the design of a transport aircraft. Any quantities not specified as outputs from a task are treated as system level inputs. The resulting system is shown in Figure 2.

Ranges of interest and baseline values for the system level inputs were selected to represent a large design space of alternatives as listed in Table 1(a).

Table 1. System level variable ranges and settings

(a) Explicit variable settings

\begin{tabular}{ccccc}
\hline \hline Quantity & Min. & Max. & Baseline & Units \\
\hline$W_{p}$ & 10,000 & 100,000 & 55,000 & $l b f$ \\
$W / S$ & 50 & 150 & 100 & $l b f / f t^{2}$ \\
$l_{f u s e}$ & 150 & 200 & 175 & $f t$ \\
$R$ & 6 & 12 & 9 & - \\
$S F C$ & 0.3 & 0.7 & 0.5 & $l b m / h r / l b f$ \\
$R$ & 3000 & 9000 & 6000 & $n m i$ \\
$W_{f, a d d} / W_{f}$ & 5 & 30 & 17.5 & $\%$ \\
$T / W$ & 0.18 & 0.3 & 0.24 & - \\
$C_{L, \max }$ & 1.0 & 4.0 & 2.5 & - \\
\hline \hline
\end{tabular}

(b) Implied variable settings

\begin{tabular}{lrl}
\hline \hline Quantity & Value & Units \\
\hline$M_{c r}$ & 0.83 & - \\
$\Lambda_{c / 2}$ & $25^{\circ}$ & \\
$D_{\text {fuse }}$ & 17 & $f t$ \\
$S_{V T} / S$ & 10 & $\%$ \\
$S_{H T} / S$ & 23 & $\%$ \\
$\lambda$ & 0.25 & - \\
$\tau$ & 12 & $\%$ \\
\hline \hline
\end{tabular}

Additionally, some quantities that would qualify as system level variables were set to representative values and held constant throughout the study. This effectively hides these quantities from the systems perspective. This was done to limit the scope of the study and the resulting visualizations to a manageable level. These implied variables and their settings are listed in Table 1(b).

The baseline settings of the system level variables result in a converged vehicle represented by the system 


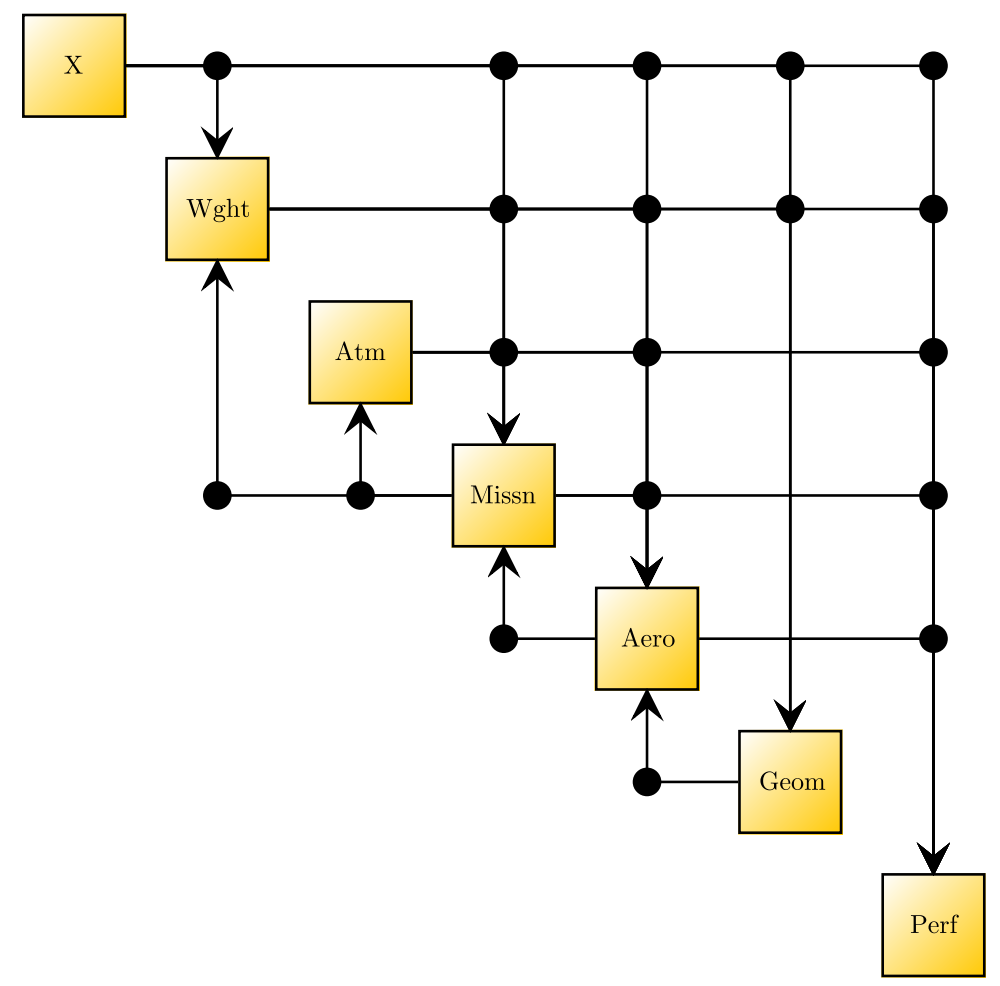

Figure 2. Example system DSM

outputs listed in Table 2(a). A three-dimensional model of the baseline aircraft is given in Figure 3.

A system explorer view of the entire aircraft design space is included as Figure 4. The hairlines represent the baseline values of the system level variables. The curves represent the variation of the system response to the change of a single system level variable. Each point contributing to a curve in the system explorer represents a converged vehicle model.

Some representative constraints were placed on the point performance of the aircraft as listed in Table 2(b). These constraints were plotted on a customary $T / W$ vs. $W / S$ view of the design space as shown in Figure 7. The vertical and horizontal hairlines represent the baseline vehicle. As expected, the baseline vehicle satisfies the constraints.

\section{Error Management}

In order to demonstrate the utility of the fidelity trade environment, a representative error management scenario is carried out on the example transport aircraft system. The focus of this example is to illustrate a possible fidelity decision making process, so the point of interest and constraint settings representing the aircraft design, and therefore the design and size of the example vehicle, will not be changed. Of course, the environment is also capable of supporting more traditional decisions in design. This scenario serves as an experiment to test the effectiveness of the fidelity trade environment, in support of testing the research hypotheses.

In this scenario, a designer is at the start of a complex systems design process using a system model based upon legacy codes. The designer is interested in accomplishing three major goals. First, choosing a baseline design for the vehicle with an understanding of the impact of error on that choice. Second, calculating a preliminary estimate of the size and performance of the baseline design with some understanding of the accuracy of those estimates. Third, determining the required analysis fidelity going forward in the design process. These fidelity decisions will guide investment and development in analysis tools. The fidelity trade environment should enable the designer to accomplish all of these goals.

The first step in the error management exercise is to introduce some representative sources of error. For problem simplicity and in order to highlight the differing impact of different error sources, the representative 
Table 2. Aircraft characteristics

(a) Sized vehicle characteristics

\begin{tabular}{lrl}
\hline \hline Quantity & Value & Units \\
\hline$W$ & 310000 & $l b f$ \\
$\sigma$ & 0.193 & - \\
$W_{f} / W$ & 34.5 & $\%$ \\
$\theta$ & 0.752 & - \\
$S_{\text {wet }, \text { wing }}$ & 5325 & $f t^{2}$ \\
$S_{\text {wet }, \text { fuse }}$ & 7800 & $f t^{2}$ \\
$S_{\text {wet }, \text { add }}$ & 2880 & $\mathrm{ft}{ }^{2}$ \\
$C_{D, 0 \mathrm{cr}}$ & 0.0151 & - \\
$C_{D, 0} \mathrm{sl}$ & 0.0113 & - \\
$e_{c r}$ & 0.735 & - \\
$e_{s l}$ & 0.655 & - \\
$S_{T O}$ & 5430 & $\mathrm{ft}$ \\
$S_{L D G}$ & 4200 & $\mathrm{ft}$ \\
$P_{s, s l}$ & 3360 & $\mathrm{ft} / \mathrm{min}$ \\
$P_{s, c r}$ & 390 & $\mathrm{ft} / \mathrm{min}$ \\
\hline \hline
\end{tabular}

(b) Aircraft constraints

\begin{tabular}{llll}
\hline \hline Quantity & & Value & Units \\
\hline$S_{T O}$ & $<$ & 6000 & $\mathrm{ft}$ \\
$S_{L D G}$ & $<$ & 4500 & $\mathrm{ft}$ \\
$P_{s, s l}$ & $>$ & 3000 & $\mathrm{ft} / \mathrm{min}$ \\
$P_{s, c r}$ & $>$ & 300 & $\mathrm{ft} / \mathrm{min}$ \\
\hline \hline
\end{tabular}$$
\text { . }
$$

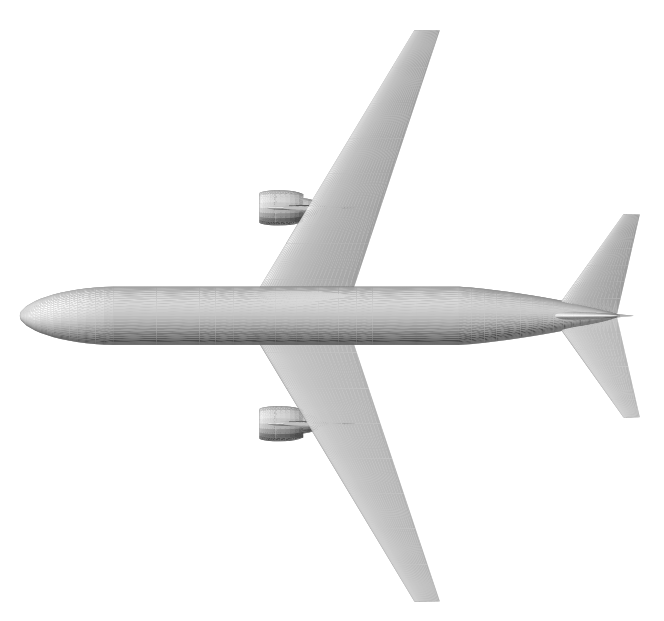

(a) Top

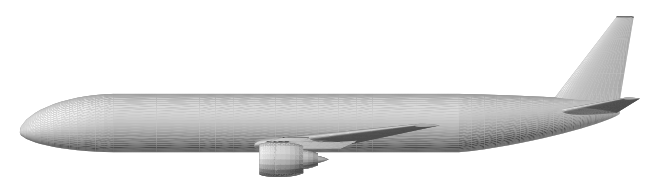

(c) Side

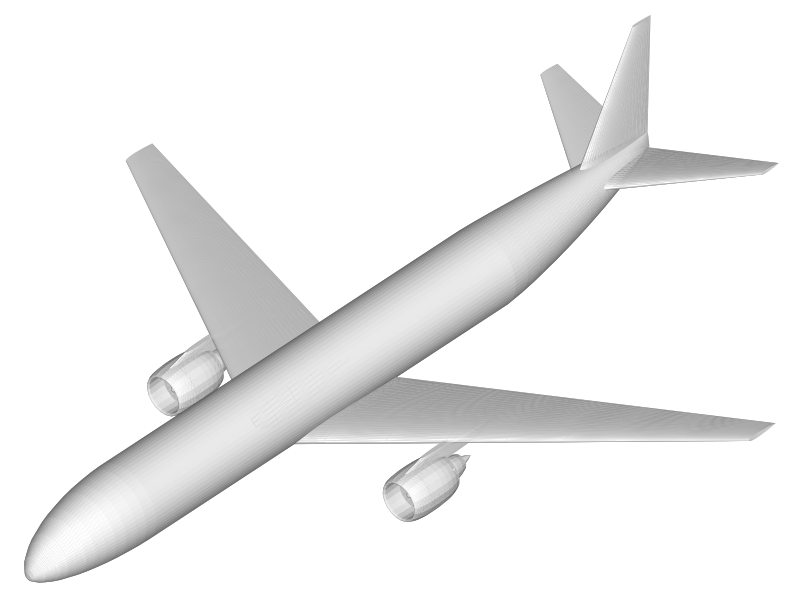

(b) Isometric

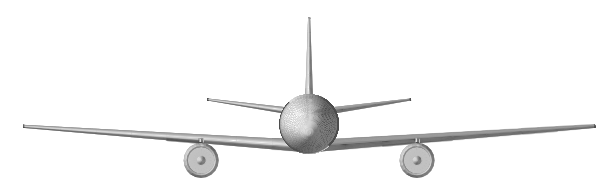

(d) Front

Figure 3. Baseline aircraft 


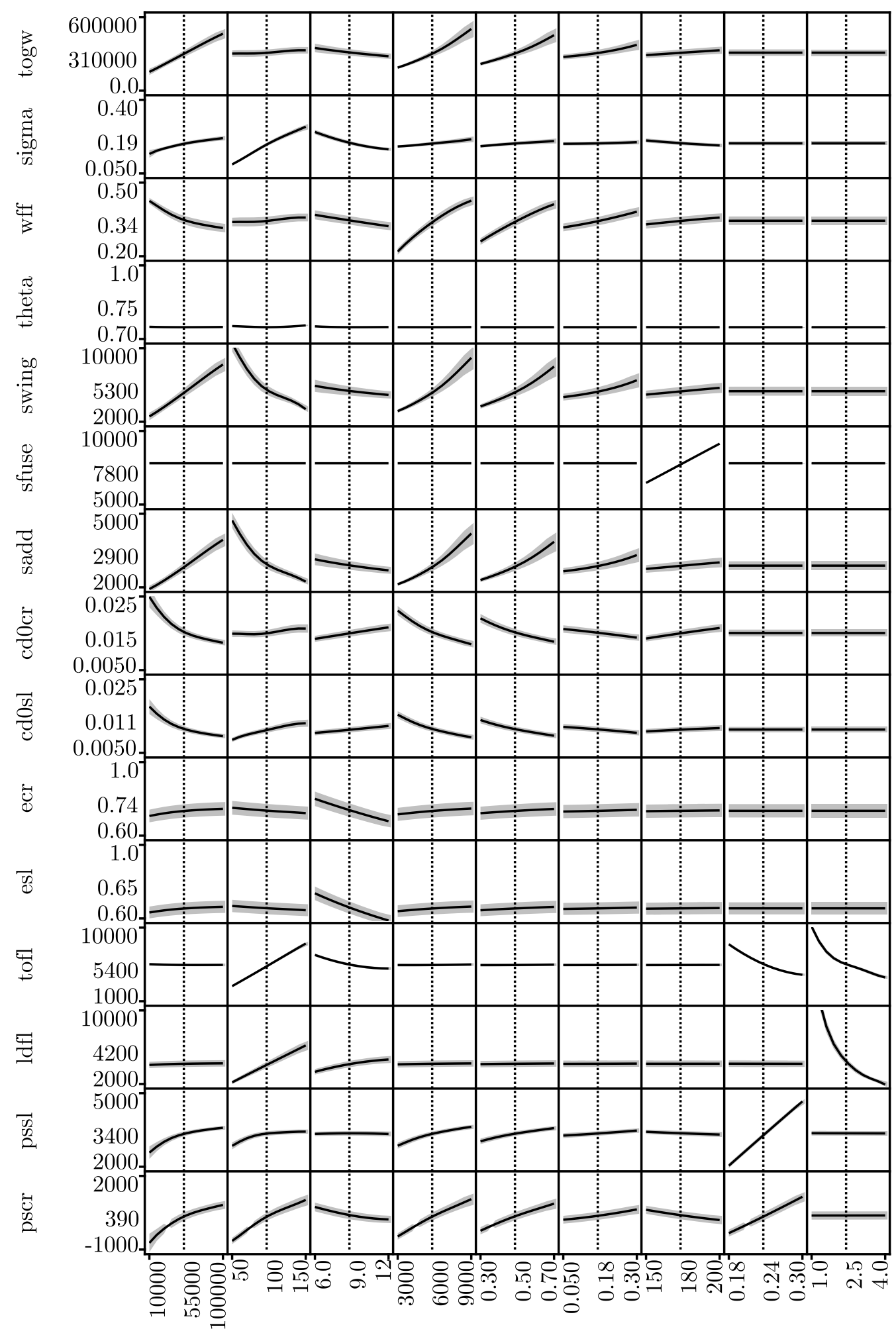

wp wos ar range sfccr wffadd lfuse tow clmax

Figure 4. Aircraft design space with introductory error 
error sources are all introduced with the same magnitude, 5\%. Similarly, when a fidelity improvement is made, the error source is reduced to $1 \%$. While these error levels are not representative of the actual error of the methods used in the example problem, they illustrate the utility of fidelity trades without introducing the complexity of making and justifying error estimates for every component. The initial error levels applied are listed in Table 3.

\begin{tabular}{cc} 
Table 3. Initial error sour \\
\hline \hline Quantity & Value \\
\hline$\sigma C_{L, \max }$ & $5 \%$ \\
$\varsigma e_{c r}$ & $5 \%$ \\
$\varsigma W$ & $5 \%$ \\
$\varsigma C_{D, 0 \mathrm{cr}}$ & $5 \%$ \\
$\sigma S F C$ & $5 \%$ \\
$\varsigma C_{D, 0} \mathrm{sl}$ & $5 \%$ \\
$\varsigma e_{s l}$ & $5 \%$ \\
$\varsigma W_{f} / W$ & $5 \%$ \\
\hline \hline
\end{tabular}

The introductory propagated error breakdown for the system variables is included as Figure 5. Specific excess power at cruise shows a very large percent error; this is largely due to the relatively small magnitude of the excess power relative to the potential changes due to the error sources.

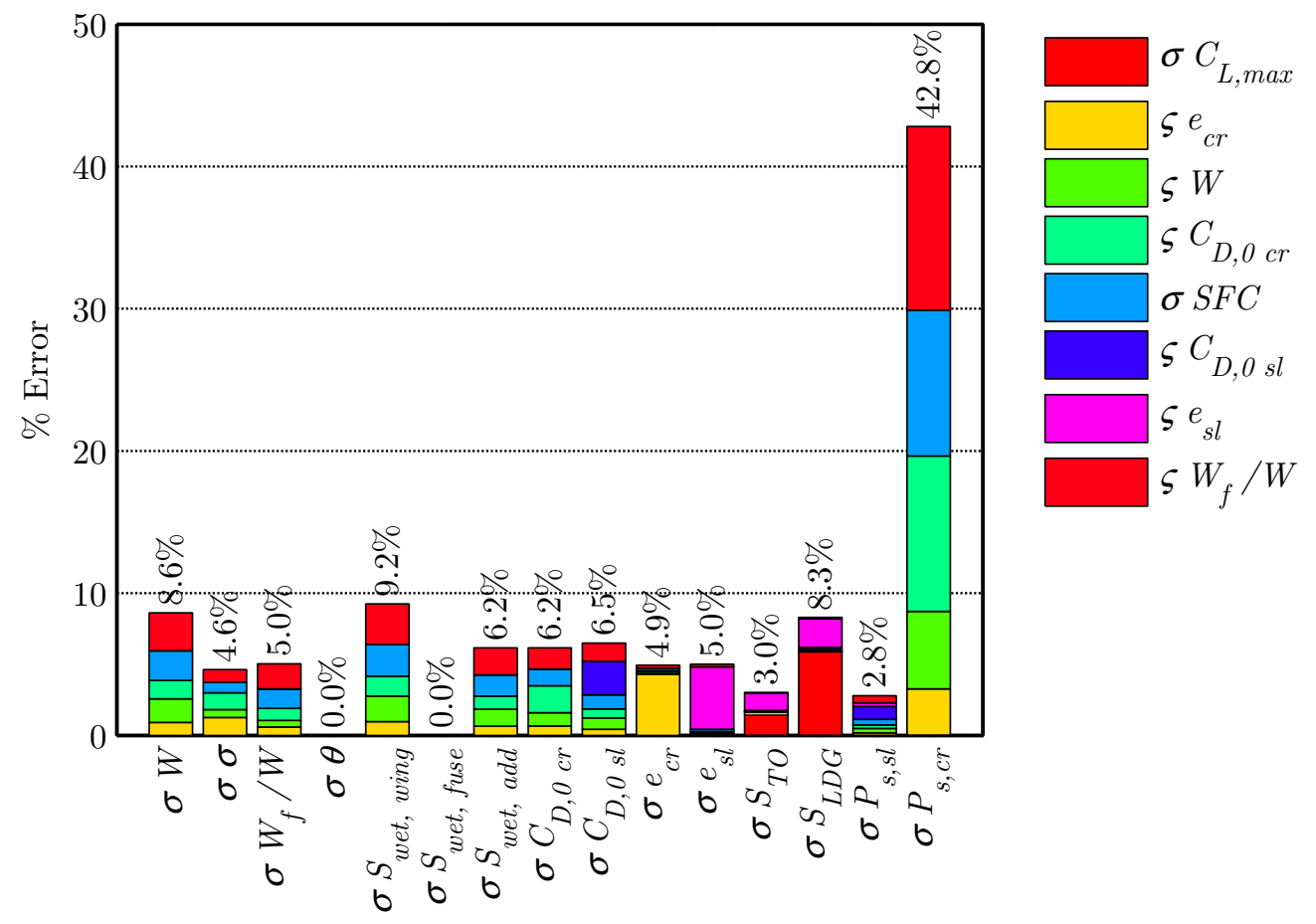

Response

Figure 5. Introductory error breakdown

As a verification experiment, a Monte Carlo approach was used to propagate the error through the complex system. The results of the Monte Carlo analysis were compared with the sensitivity based approach in Figure 6. The blue and red bars represent the results of the sensitivity approach and the Monte Carlo approach respectively. The small error bands at the top of the Monte Carlo bar represent the one sigma error in the result due to the use of a finite sample size.

For the Monte Carlo study, Gaussian probability distributions with zero mean and the specified variance 


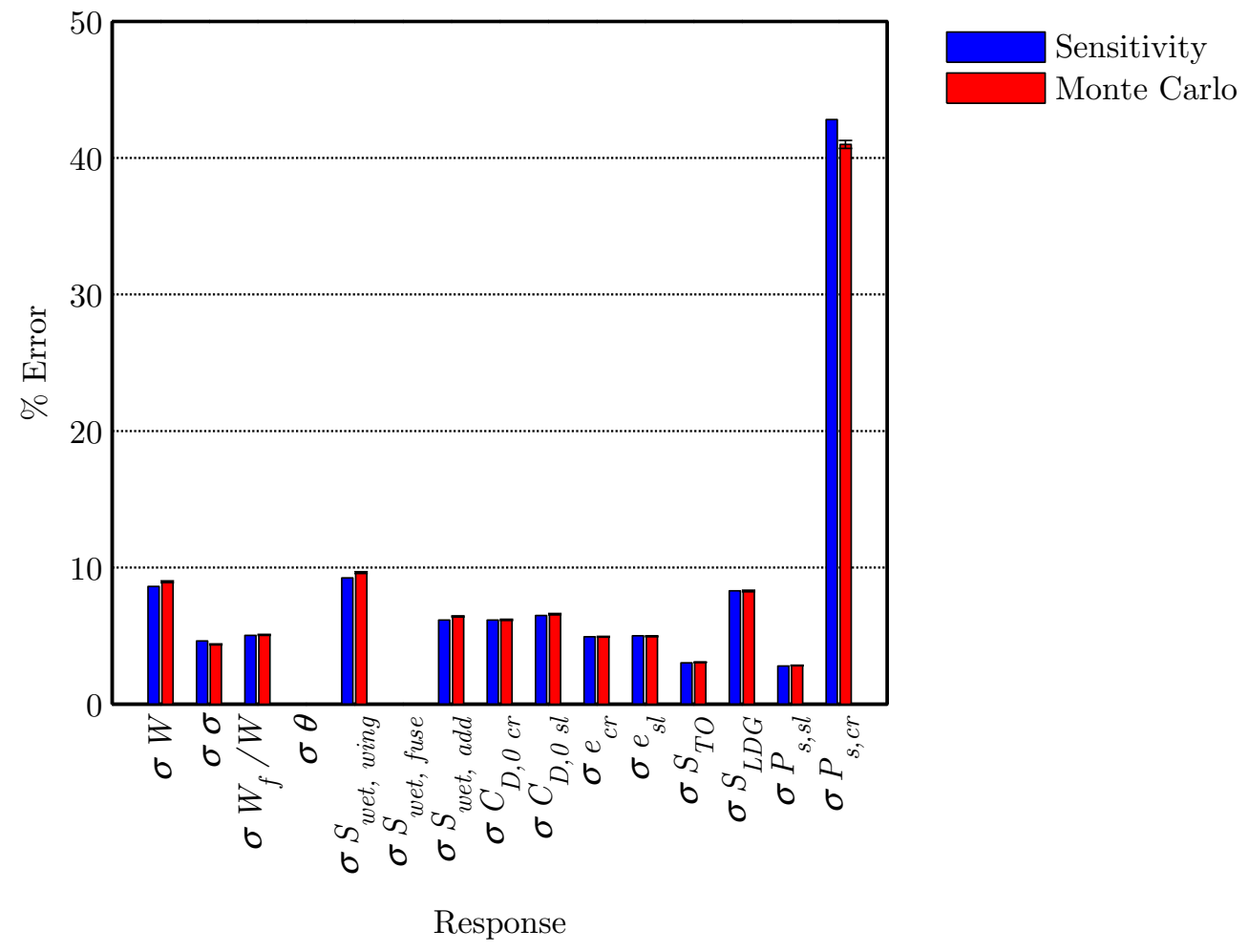

Figure 6. Introductory error verification

were assumed for each of the error sources. A random sample of 10,000 cases were analyzed, and the complete system was brought to convergence for every case.

In general, the agreement between the two approaches is very good, and the sensitivity approach is verified as an approximate model of the propagation of error through a complex system. The $\sigma P_{s, c r}$ result shows the greatest disparity, clearly beyond that which can be attributed to the finite sample size; this disparity is due to the linearization introduced by the sensitivity approach.

The Monte Carlo verification analysis took about five minutes to complete for a single point in the design space. Each update of the fidelity trade environment would require approximately 500 such analyses. The sensitivity approach allows what would otherwise be a very expensive calculation to be performed in an interactive manner, thereby making the fidelity trade environment possible. The comparative nature of the results displayed by the fidelity trade environment do not require extreme accuracy, and the approximations introduced by the linearization are believed by the author to be acceptable.

The gray band surrounding the system response in Figure 4 represents the system propagated error throughout the design space. The impact of error on the design space as represented by the constraint diagram is shown in Figure 7. Note that the error on the landing field length constraint has dramatic impact on the choice of the design point of the vehicle. Not only have the constraint locations with the consideration of error changed, but the constraints active in choosing a design point have changed. Before, the design point was constrained by specific excess power at cruise and takeoff field length; afterward, the design point was constrained by specific excess power at cruise and landing field length.

When faced with the constraint diagram depicted in Figure 7, the designer may desire to reduce the error impacting his choice of design point. His first step may be to improve the landing field length estimation. Referring to Figure 5, he notices that the primary source of error for landing field length is the error in maximum lift coefficient. The designer may then choose to perform a wind tunnel test to reduce the error on this quantity.

Further examination of Figure 7 demonstrates that the impact of error on the design point would then be primarily due to error in specific excess power at cruise. Reducing this error would be the designer's priority. The error breakdown shown in Figure 5 indicates which error sources have the greatest impact on 


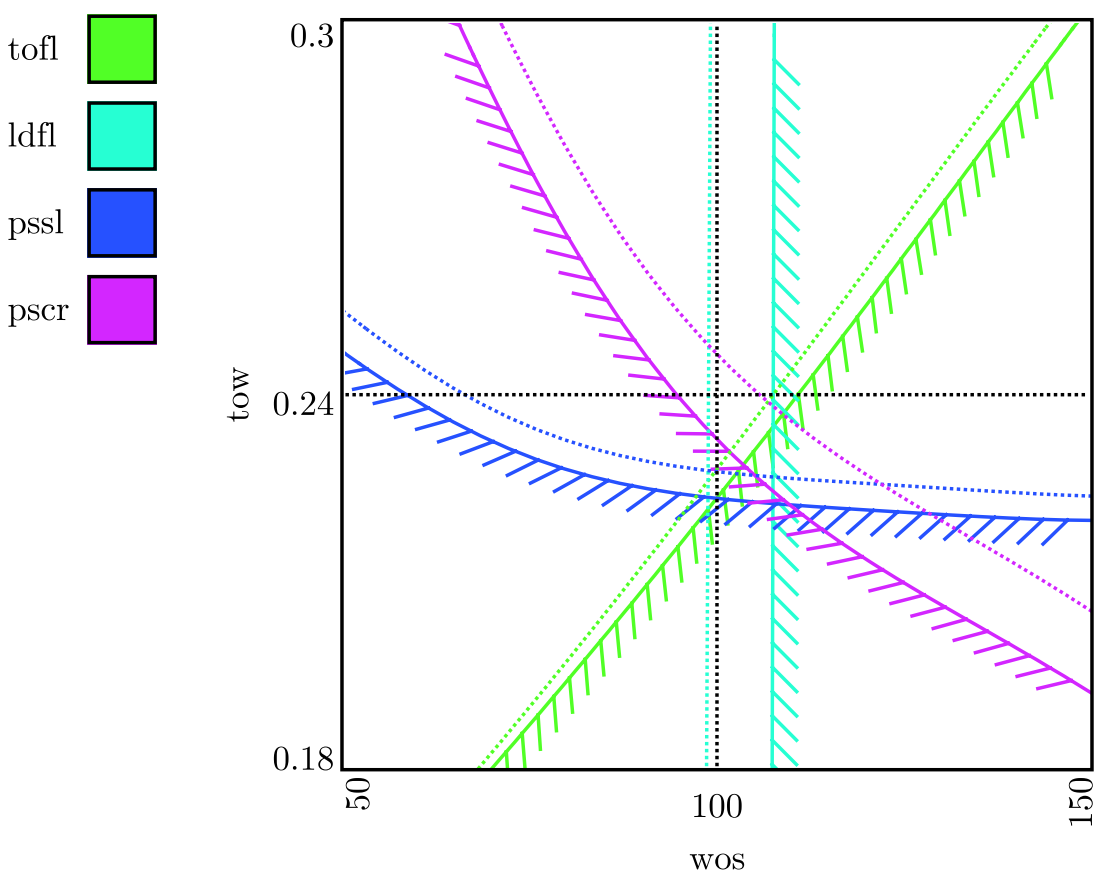

Figure 7. Aircraft constraints with introductory error

cruise specific excess power. The cruise drag coefficient estimate $C_{D, 0} \mathrm{cr}$ could be improved through a wind tunnel test, while an improved estimate of the cruise specific fuel consumption $S F C$ could be requested from the engine manufacturer. An improved mission model could improve the fuel burn calculation $W_{f} / W$.

Some representative reduced error levels for the system are listed below in Table 4 .

Table 4. Reduced error levels

\begin{tabular}{ll}
\hline \hline Quantity & Value \\
\hline$\sigma C_{L, \max }$ & $1 \%$ \\
$\varsigma e_{c r}$ & $5 \%$ \\
$\varsigma W$ & $5 \%$ \\
$\varsigma C_{D, 0 \mathrm{cr}}$ & $1 \%$ \\
$\sigma S F C$ & $1 \%$ \\
$\varsigma C_{D, 0} s l$ & $5 \%$ \\
$\varsigma e_{s l}$ & $5 \%$ \\
$\varsigma W_{f} / W$ & $1 \%$ \\
\hline \hline
\end{tabular}

The error breakdown corresponding to the system for reduced error is shown in Figure 8. Note that the error in takeoff and landing distance were significantly reduced. While still showing the largest percent error, error in cruise excess power has been reduced enough to warrant changing the scale of the error breakdown.

Figure 9 demonstrates the effectiveness of reducing the error on the design point selection. The active constraints have changed and the impact of error on choosing the design wing loading has been minimized.

Interpretation of Figure 9 may show that the level of error impacting the design point is acceptable to the designer. Once a design point is selected, the primary purpose of an aircraft systems study is to estimate the size of the vehicle. The takeoff gross weight of an aircraft is a primary driver of the manufacturing, operating, maintenance, and other costs of the vehicle. In the absence of further analysis, minimization of aircraft weight tends to minimize cost. Examination of the error breakdown shown in Figure 8 shows that the biggest remaining contributor to error in takeoff gross weight is the weight calculation itself. The designer may then choose to reduce the error in the weight calculation in some manner.

In this scenario, the designer was faced with a complex system incorporating a variety of error sources. 


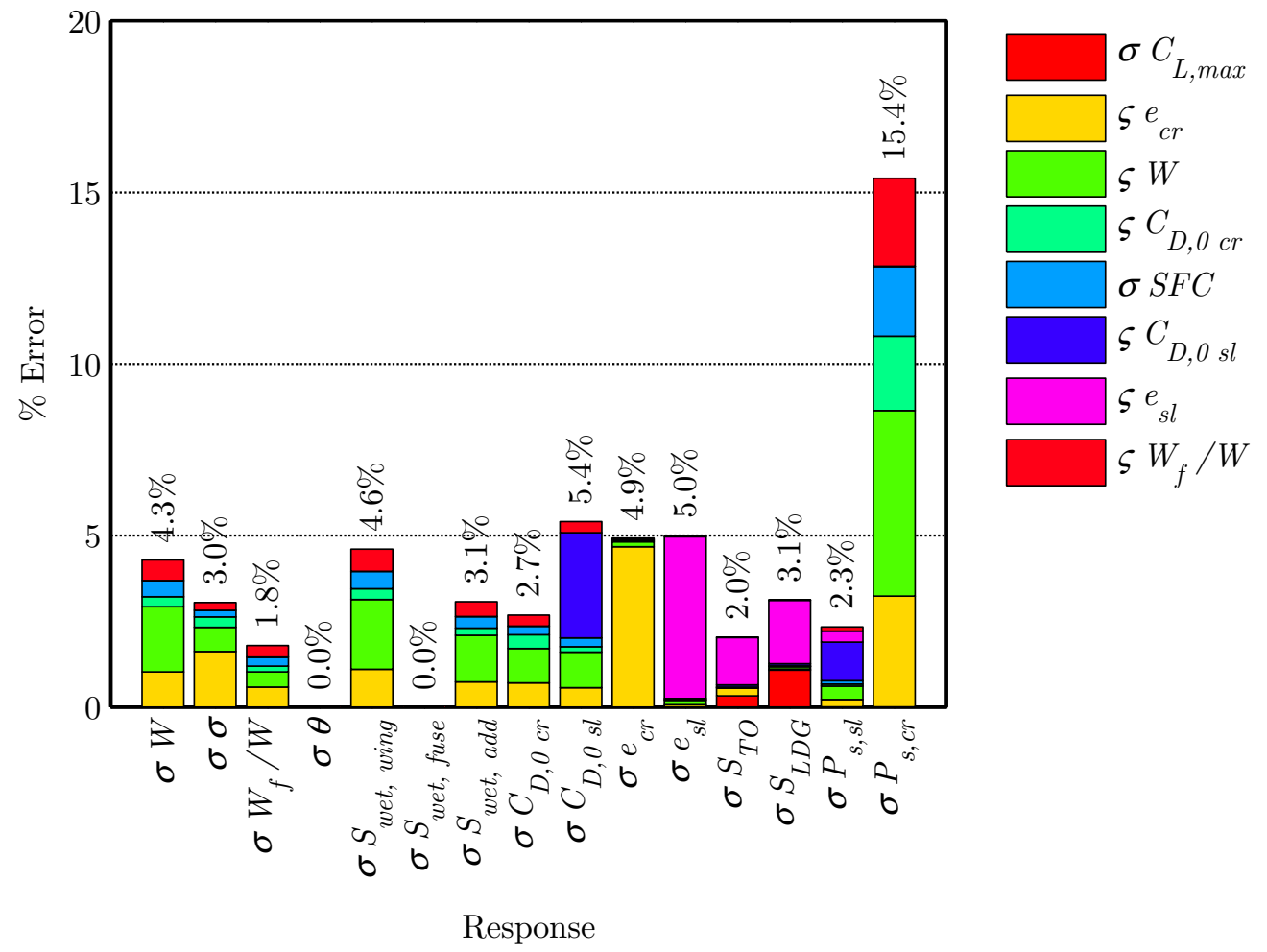

Figure 8. Error breakdown with reduced error
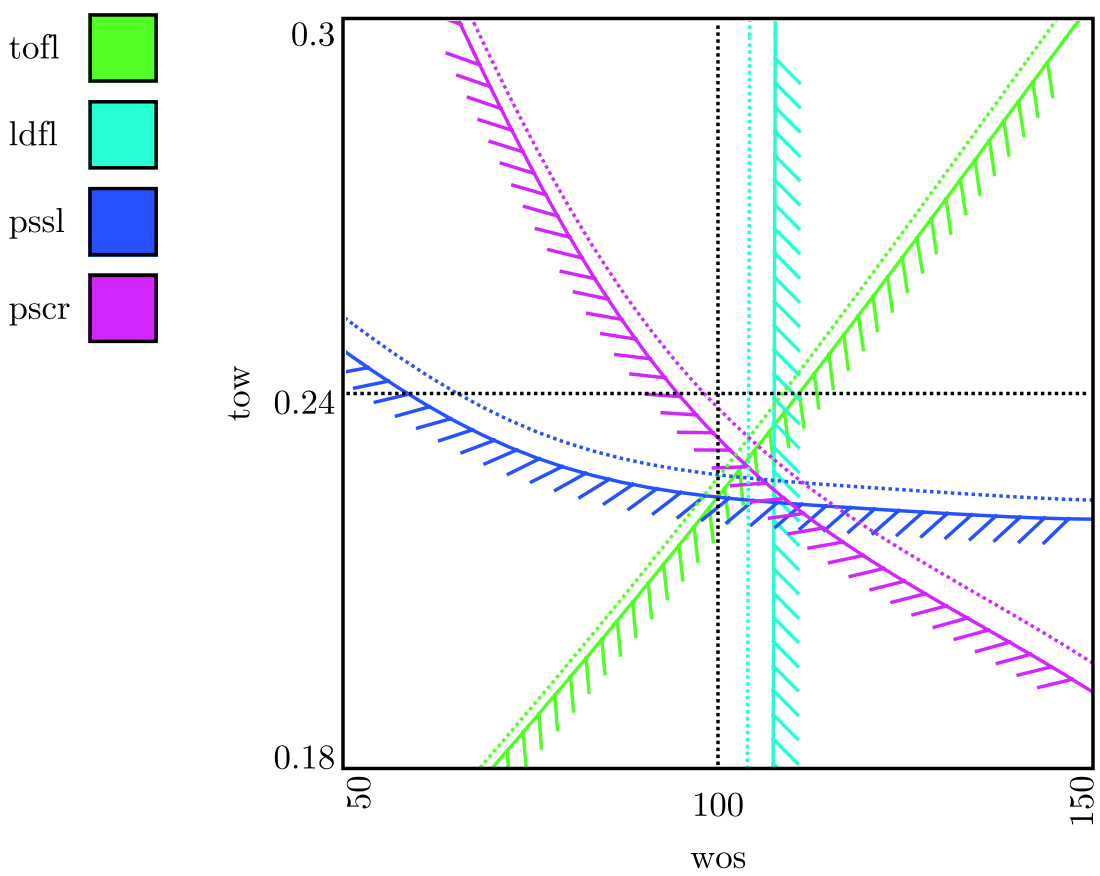

Figure 9. Aircraft constraints with reduced error 
His first actions were to reduce the sources of error contributing to uncertainty in the choice of the design point. Once a design point could be chosen with confidence, his next action was to reduce the sources of error contributing to the most important system level metrics. In so doing, some significant sources were allowed to remain, being quantitatively determined to be unimportant to the decision making process undertaken by the designer.

\section{Conclusions}

This paper described the conception, formulation, and creation of a fidelity trade environment. The fidelity trade environment was used to model a representative complex system. Successful decision making processes concerning the fidelity of tools in complex systems design studies were demonstrated; this scenario was not possible without the fidelity trade environment.

This research has developed the fidelity trade environment as a decision making tool to help the designer answer questions like: Is the fidelity of a complex systems analysis adequate, or is an improvement needed? If an improvement is needed, how is that improvement best achieved? Where should limited resources be invested for the improvement of fidelity? How does knowledge of the imperfection of a model impact design decisions based on the model? How does this knowledge impact the choice and certainty of the design point? How does it impact the certainty of the performance of a particular design?

\section{References}

\footnotetext{
${ }^{1}$ Roache, P. J., Verification and Validation in Computational Science and Engineering, Hermosa Publishers, Albuquerque, New Mexico, 1998.

${ }^{2}$ Baird, D. C., Experimentation: An Introduction to Measurement Theory and Experiment Design, Prentice-Hall Inc., Englewood Cliffs, NJ, 1962.

${ }^{3}$ Taylor, J. R., An Introduction to Error Analysis The Study of Uncertainties in Physical Measurements, University Science Books, Sausality, California, 2nd ed., 1997.

${ }^{4}$ Oberkampf, W. L., Diegert, K. V., Alvin, K. F., and Rutherford, B. M., "Variability, Uncertaintity, and Error in Computational Simulation," HTD-Vol. 375-2, ASME Proceedings of the 7th AIAA/ASME Joint Thermophysics and Heat Transfer Conference, edited by R. S. Amano, B. F. Armaly, T. S. Chen, A. Emery, J. A. Liburdy, D. P. Shatto, N. K. Anand, B. Blackwell, T. Y. Chu, J. Lage, O. Oosthuizen, and K. A. Woodbury, No. H1137B, 1998.

${ }^{5}$ Oberkampf, W. L., DeLand, S. M., Rutherford, B. M., Diegert, K. V., and Alvin, K. F., "Error and Uncertainty in Modeling and Simulation," Reliability Engineering \& System Safety, Vol. 75, 2002, pp. 333-357.

${ }^{6}$ SAS Institute, JMP User Guide, Release 6, Cary, NC, 2005.

${ }^{7} \mathrm{Du}, \mathrm{X}$. and Chen, W., "Efficient Uncertainty Analysis Methods for Multidisciplinary Robust Design," AIAA Journal, Vol. 40, No. 3, 2002, pp. 545-552.

${ }^{8}$ Sobieszczanski-Sobieski, J., "Sensitivity of Complex, Internally Coupled Systems," AIAA Journal, Vol. 28, No. 1, January 1990, pp. 153-160.

${ }^{9}$ Sobieszczanski-Sobieski, J., "Higher Order Sensitivity Analysis of Complex, Coupled Systems," AIAA Journal, Vol. 28, No. 4, April 1990, pp. 756-758.

${ }^{10}$ Steward, D. V., "The Design Structure System: A Method for Managing the Design of Complex Systems," IEEE Transactions on Engineering Management, Vol. EM 78, No. 3, August 1981, pp. 71-74.

${ }^{11}$ McDonald, R. A., Error Propagation and Metamodeling for a Fidelity Tradeoff Capability in Complex Systems Design, Ph.D. thesis, Aerospace Engineering, Georgia Institute of Technology, Atlanta, GA, August 2006.

${ }^{12}$ Phoenix, Improving The Engineering Process With Software Integration; Integrating Engineering Applications for Design, Phoenix Integration, Blacksburg, VA, February 2003.
} 